\title{
Reflets
}

Revue ontaroise d'intervention sociale et communautaire

\section{L’évolution de la profession de sage-femme}

\section{Nathalie Mélanson}

Volume 5, numéro 2, automne 1999

La santé des francophones de l’Ontario

URI : https://id.erudit.org/iderudit/026283ar

DOI : https://doi.org/10.7202/026283ar

Aller au sommaire du numéro

Éditeur(s)

Reflets : Revue ontaroise d'intervention sociale et communautaire

ISSN

1203-4576 (imprimé)

1712-8498 (numérique)

Découvrir la revue

Citer cet article

Mélanson, N. (1999). L'évolution de la profession de sage-femme. Reflets, 5(2), 247-255. https://doi.org/10.7202/026283ar

Tous droits réservés (C) Reflets : Revue ontaroise d'intervention sociale et communautaire, 1999
Ce document est protégé par la loi sur le droit d'auteur. L'utilisation des services d'Érudit (y compris la reproduction) est assujettie à sa politique d'utilisation que vous pouvez consulter en ligne.

https://apropos.erudit.org/fr/usagers/politique-dutilisation/ 


\section{L'évolution de la profession de sage-femme}

\section{$N$ athalie M élanson}

N ous sommesau seuil de l'an 2000, ère technologique fascinante et fantastique, mais sussi ère de grandes contradictions. D 'un côté, nous pouvons maintenant guérir certaines formes de cancer, être vaccinés contre certaines maladies mortelles, repousser de plusen plus l'heure de notre mort et contrôler pratiquement tous les aspects de la naissance, de la sélection génétique àl'accouchement programmé. De l'autre, nous réclamons un retour à la nature, au respect du corps et de son infinie sagesse pour savoir ce qu'il a à faire. En effet, c'est depuis les derniersvingt ansque se multiplient les diverses médecines douces et les activités naturalistes comme I'homéopathie, la naturopathie, le yoga, la méditation, etc. Et la profession de sage-femme fait partie intégrante de ce courant.

$D$ ans les pages qui suivent, nous brosserons un tableau de cette profession telle qu'elle existe en 0 ntario, en donnant d'abord un bref historique de sa récente renaissance, pour ensuite la présenter dans le contexte présent: son état actuel, ses principes de base, la formation des sages-femmes, les relations entre elles et le corps médical. N ous terminerons avec nos perspectives d'avenir pour la profession, en $\mathrm{O}$ ntario et dans le reste du pays.

\section{Une renaissance}

Selon toute logique, on peut dire que la plus ancienne des professions est celle de sage-femme, tout simplement parce que les femmes accouchent depuis le début des temps et que l'on a des preuves à l'effet que lors de l'accouchement, les femmes ont toujours été aidées par d'autres femmes(C ochrane 1995). C e n'est 
que depuis une centaine d'années que le corps médical s'est approprié le monopole dans ce domaine, pour diverses raisons sociales, politiques et économiques. C'est ainsi qu'on en est arrivé à médicaliser et à dénaturer le processus de la naissance, tout particulièrement en A mérique du N ord (Arms 1975; Saillant et 0 'N eil 1987; D avis-Floyd 1992; M itford 1992).Ainsi, la majorité des sages-femmes nord-américaines a été discréditée, au point de disparaître presque complètement.

Par contre, depuis quelques décennies, sous l'influence du mouvement de la contre-culture américaine des années 1960 et celle du mouvement féministe des années 1970, les femmes réclament de plus en plus leurs droits. Plus particulièrement, elles réclament des soins périnataux plus personnalisés, moins interventionnistes et plus féminisés, ainsi que l'accouchement à domicile, où elles participent plus activement aux prises de décisions qui les concernent. Bien que le milieu obstétrical ait connu beaucoup de changements positifs depuis l'époque de I'utilisation routinière des forceps, des étriers, de l'épisiotomie et de l'anesthésie générale, il n'en reste pas moinsque pour plusieurs femmes, les sages femmes ont répondu à leurs besoins, puisque le nombre de sages-femmes s'est accru depuis vingt ans et, plus particulièrement, au cours des cinq dernières années en 0 ntario (O ntario M inistry of $\mathrm{H}$ ealth 1987b;Tyson et al. 1995).

A u C anada, les sages-femmes ont longtemps œuvré sans statut légal, tout en luttant ardemment pour légaliser la profession et pour rendre leurs services accessibles à toutes les femmes. C ette situation d'illégalité persiste d'ailleurs dans la plupart des provinces canadiennes, l'O ntario étant la première à avoir légalisé la profession. En effet, en 1991, après plus de dix ans de revendications, le gouvernement ontarien a réglementé la profession de sage-femme et la loi est entrée en vigueur en 1994. Les autres provinces canadiennesen sont à divers stades. Le Q uébec a présenté son projet de loi à l'été 1999 et l'U niversité du Q uébec àTrois$R$ ivières a accueilli sa première cohorte d'étudiantes sages-femmes en septembre 1999. La profession est légalisée en Alberta, mais elle n'est pas complètement financée par le gouvernement (G overnment of A lberta N ewsR elease 1999). Il reste à mettre en 
place un programme de formation en Colombie-Britannique (C lelland 1999). Q uant aux autres provinces, elles en sont encore aux stades préliminaires: soit que la profession est réglementée, mais on attend encore la mise en vigueur du projet de loi, soit qu'on en est encore à l'étape de l'élaboration du projet, soit qu'aucune initiative n'a encore été prise ( $\mathrm{N}$ ewfoundland and Labrador M idwives'A ssociation 1999). Le processus est plus long dans certaines provinces, mais nous espérons qu'éventuellement les services de sages femmes seront légalement disponiblesà travers le Canada et qu'ils seront entièrement financés par le gouvernement.

\section{Devenir sage-femme}

Aujourd'hui, les sages-femmes ontariennes sont autorisées à pratiquer soit parce qu'elles ont suivi un programme de formation universitaire ou parce qu'elles ont traversé le processus d'accréditation de l'O rdre des sages-femmes de I'O ntario (O SFO ). Ce processus ne s'adresse qu'à celles qui pratiquaient déjà au moment de l'entrée en vigueur de la loi régissant la profession (certification par le biais de l'Institut M itchener deToronto) ou qui ont été formées dans d'autres pays (Prior Learning and Experience Assessment (PLEA) de l'O SFO ). Le programme de formation des sages-femmes de l'O ntario, qui existe depuis septembre 1993, offre un baccalauréat de quatre ans en sciences de la santé (profession de sage- femme). C elui-ci est offert par un consortium de troisuniversités ontariennes: I'U niversité M CM aster à $\mathrm{H}$ amilton offre la formation en anglaisà tempsplein, I'U niversité Laurentienne de Sudbury offre la formation en français à temps plein et I'U niversité Polytechnique $R$ yerson offre la formation en anglais à temps partiel (sur une période de cinq à sept ans) (O rdre des sages-femmes de l'O ntario 1994). En créant le programme, les sages-femmes se sont inspirées des modèles de pratique de divers pays, incluant ceux qui sont offerts aux États$\mathrm{U}$ nis, en $\mathrm{G}$ rande- $\mathrm{B}$ retagne, au $\mathrm{D}$ anemark et en $\mathrm{H}$ ollande $\mathrm{O}$ ntario $M$ inistry of $\mathrm{H}$ ealth 1987a). La formation comprend des cours en sciencesfondamentales, en sciencessociales, en sciences de la santé, en études de la femme, ainsi que des cours cliniques et des cours 
au choix (O rdre des sages-femmes de l'O ntario 1994). À la suite des recommandations du $C$ urriaulum $D$ esign $C$ ommittee, au moins la moitié de la formation comprend de l'expérience clinique. En effet, six des neuf trimestres du programme sont passés en milieu clinique afin que les étudiantes puissent apprendre dans le cadre du modèle de pratique des sages femmes(Tyson et al. 1995). Enfin, lorsqu'elle reçoit son diplôme, une étudiante sage-femme aura accompagné au moins 60 parturientes, dont 40 en tant que sagefemme responsable de l'accouchement et 30 accouchements (au moins dix à l'hôpital et dix à domicile ou dans un centre d'accouchement) où elle assure la continuité des soins durant toute la grossesse: période prénatale, accouchement et post-partum (O rdre des sages-femmes de l'O ntario 1994). Ainsi, elle aura participé activement à toutes les étapes de la grossesse, de l'accouchement à de la période post-partum et ce, dans tous les milieux.

Les étudiantes sages-femmes proviennent de divers milieux. Alors que certaines font un passage direct entre les études secondaires et universitaires, d'autres ont déjà effectuées quelques années d'études universitaires ou ont déjà obtenu diplôme, voire deux et, finalement, d'autres encore retournent aux études après avoir élevé leur famille ou après avoir œuvré dansun autre domaine professionnel. Cette diversité d'expériences constitue un atout important pour le programme et la profession, car chacune de ces femmes y apporte son vécu.

\section{Être sage-femme}

Les sages-femmes ontariennes sont autorisées à assister à des accouchements à domicile, dans des hôpitaux et dans des centres d'accouchement (Tyson et al. 1995). Elles sont salariées et pratiquent indépendamment des médecins dans des cliniques subventionnées par le ministère de la Santé. U ne cliente n'a donc pas à débourser de frais pour les services d'une sage-femme. Selon les normes de pratique établies par l'O rdre des sages-femmes de I'O ntario, une sage-femme qui pratique à plein temps, assiste à au moins 80 accouchements par année, soit 40 en tant que sage- 
femme responsable et 40 en tant que soutien ( 0 rdre des sagesfemmes de l'O ntario 1994). C ette pratique et le fait d'être salariée plutôt que rémunérée selon un nombre de clientes, lui permet d'assurer un soin personnalisé de qualité à chaque femme.

\section{Les soins de sage-femme}

Selon la L oi de 1991 sur les professions de la santé réglementées et conformément à la définition internationale de la sage-femme:

L'exercice de la profession de sage femme consiste à évaluer et à surveiller les femmes pendant la grossesse, I' accouchement et la suite de couches, ainsi qu'à évaluer, à surveiller leur nouveau-né, à dispenser des soins pendant une grossesse normale, un accouchement normal et la suite de couches normale, et à pratiquer des accouchements normaux et spontanés par voie vaginale (O rdre des sages-femmes de l' 0 ntario 1994).

Les sages-femmes de l'O ntario ne s'occupent donc que des cas normaux de grossesse et d'accouchement et elles travaillent de concert avec les médecins, soit en leur transférant certaines clientes nécessitant leur expertise, soit en les consultant pour certains cas.

À l'instar des revendications des femmes face à la surmédicalisation de la naissance et de leurs réclamations pour des soins de santé, les trois principes de base suivants ont été établis pour la profession: la continuité des soins, le choix éclairé et le choix du lieu d'accouchement. La continuité des soinssignifie qu'une femme recevra les soins d'un groupe de quatre sagesfemmes, au maximum, au cours de sa grossesse, son travail, son accouchement et les six semaines suivant celui-ci. Sa sage-femme primaire devra demeurer disponible en tout temps de la journée, afin de pouvoir répondre à ses questions et à ses inquiétudes. À son accouchement, la cliente aura la présence d'au moins deux personnes qu'elle aura connues au cours des soins prénataux, soit deux sages-femmes ou, le cas échéant, une sage-femme et une auxiliaire ayant de l'expérience dans le domaine de l'accouchement. 
Le choix éclairé est « un processus décisionnel qui se fie sur l'échange d'information dansun milieu détendu, non autoritaire et en toute collaboration » ( 0 rdre des sages-femmes de l'O ntario 1994). C ela signifie qu'avant de faire un choix, la cliente recevra toute l'information qu'il lui faut sur les avantages et les risques possibles concernant toute procédure (échographie, lieu d'accouchement, médicaments, déclenchement de travail, etc.) et ce, afin de lui permettre de faire un choix éclairé. En dernier ressort, le choix de la cliente devra être respecté. C et échange d'information peut se faire lors des cours prénataux offerts par les sages-femmes ou lors de rendez-vous à la clinique. C'est d'ailleurs un des buts des visites prénatales et postnatales dont la durée varie de 45 à 60 minutes. Ainsi, la cliente peut participer activement aux décisionsqui la concernent. Par ailleurs, la relation entre la sage-femme et sa cliente ne connaît pas les écarts hiérarchiques et autoritaires caractéristiques du milieu médical, ce qui permet une relation plus égalitaire.

Enfin, et dans la mesure du possible, la cliente a le droit de choisir le lieu de son accouchement: à domicile, à l'hôpital ou dans un centre d'accouchement (là où il y en a) ( 0 rdre des sagesfemmes de l'O ntario 1994).

\section{Main dans la main avec les médecins...}

En ce qui concerne les médecins, il est vrai de dire qu'historiquement, les relations entre les sages femmes et le corps médical ont toujours été plutôt tendues, ces derniers ayant acquis le monopole dans le domaine de l'obstétrique ( 0 ntario $M$ inistry of $\mathrm{H}$ ealth 1987b). Par contre, les services des sages-femmes ontariennes sont intégrés au système de santé, ce qui entraîne des relations qui s'améliorent toujours, au fur et à mesure que la profession de sage-femme est démystifiée et que les médecins et les infirmières ont l'occasion de travailler avec elles, de voir et de comprendre comment elles pratiquent.

En 0 ntario, au moment de la réglementation de la profession, les membres de l'O rdre des obstétriciens et des gynécologues du $C$ anada ont annoncé qu'ils croyaient que cela serait très bénéfique pour les femmes enceintes. Q uant à l'A ssociation des médecins 
de l'O ntario, celle-ci n'appuyait pas l'introduction des sagesfemmes et elle croyait que les problèmes concernant les soins de maternité pourraient être solutionnés en faisant des changements au sein du corps médical (Kaufman 1991). En pratique, actuellement, les attitudes des médecins envers les sages-femmes reflètent toujours ces deux positions. A lors que les uns appuient complètement la profession, d'autres ne le font pas et certains demeurent indécis. Pourtant, dans les cinq dernières années, la situation s'est améliorée parce que les sages-femmes ont fait leurs preuves. Q ui sait, avec le temps, les sages-femmes et les médecins apprendront-ilsà travailler de concert et en toute harmonie? Enfin, on pourrait aussi espérer que dans l'avenir, Iorsqu'il y aura suffisamment de sages-femmes pour répondre à la demande, la majorité des cas normaux de grossesses seront suivis par celles ci, collaborant harmonieusement avec les obstétriciens qui s'occuperont, eux, des cas problématiques. En effet, avec une clientèle allégée, ces derniers seront davantage en mesure d'offrir des soins de qualité. $D$ 'ailleurs, avec la pénurie que l'on connaît actuellement dans le domaine médical, les sages-femmes constituent une solution efficace au problème de l'obstétrique.

\section{Une profession florissante}

La popularité grandissante des sages femmes et la pénurie de médecins spécialistes que connaissent toutes les régions, dont les obstétriciens, il est évident que les sages-femmes ont un avenir très fructueux en 0 ntario. II n'aura suffit que cinq ans, au début desquels nombre de gens étaient encore sceptiques à l'idée d'accoucher avec une sage-femme, pour que la profession se bâtisse une excellente réputation. Celle-ci s'est d'ailleurs construite principalement par le bon vieux «bouche à oreille »: une mère ravie en fait les éloges à ses sœurs, ses voisines, ses amies, qui toutes demandent alors les mêmes services! À l'heure actuelle, les femmes sont de plus en plus nombreuses à retenir ou à désirer les soins offerts par les sages femmes.

En 1994, on comptait environ 70 sages femmes inscrites avec I'O rdre des sages-femmes de l'O ntario.A ujourd'hui, on en compte 154, plus du double en cinq ans, réparties dans une quarantaine 
de cliniques. Et ce nombre ne suffit pas à répondre à la demande actuelle. II faudra patienter encore quelques années avant que toutes les régions de l'O ntario soient desservies. O n prévoit qu'environ 30 ou 40 nouvelles sages-femmes seront accréditées par année, par le biais du programme de formation universitaire ou du PLEA. Ceci signifie qu'au bout d'un autre cing ans, on pourrait avoir jusqu'à 350 sages-femmes pratiquantes.

Le programme de formation des sages-femmes est très contingenté. $\mathrm{N}$ on seulement ne peut-on pas accepter plus d'étudiantes qu'il y a de places disponibles dans les cliniques pour les former, mais on doit aussi donner le temps au gouvernement d'intégrer toutes ces sages-femmes à la fois dans des pratiques existantes ou de nouvelles pratiques, dans son budget et, bien entendu, dans la mentalité collective. II faudra donc attendre encore quelques années avant que ces services ne soient disponibles partout dansla province. À l'exception de la région métropolitaine deToronto qui a une douzaine de cliniques, les services des sagesfemmes sont moins faciles à obtenir dans la plupart des autres régions, tout particulièrement dans le $\mathrm{N}$ ord qui n'a que cinq cliniques (Sudbury,T hunder Bay, Sault Ste-M arie, Little C urrent, Powassin). Toutefois, la situation s'améliore, car on réclame de plus en plus de sages-femmes dans toutes les régions de la province. Par conséquent, dans les prochaines années, on devrait réussir à ouvrir des cliniques partout dans la province. Enfin, avec le progrès du dossier dans les autres provinces, on peut souhaiter que le jour vienne où toutes les femmes auront le choix d'obtenir les soins de sage-femme où qu'elles soient au $C$ anada.

\section{Bibliographie}

AR M S, S. (1975). I mmaculate D eception, N ew York, Bantam Books.

CLELLAN D, D. (1999, mars), «M idwifery Taskforce Journal », The M idwifery Task Force of BC, Disponible : U R L : http:/ / www.mtf.bc.cal new slett.htm\#journal

COCHR ANE, S. (1995), «M idwives, nurses and doctors: interprofessional relationship » dansT. M urphy- Black (dir.) Issues in M idwifery, L ondon, C hurchill, p. 254-9.

DAVIS-FLOY D, R . (1992) Birth as an A merican R ite of Passage, Berkeley, U niversity of California Press. 
GOU VER N EM EN T DE L'ALBERTA (1999, 6 mai). «M idwifery receives additional start-up funding », G overnment of A lberta N ews R elease, D isponible : U R L : http:// www.gov.ab.ca/ acn/ 199905/ 7626.html

KAU FM AN , K. (1991) «The introduction of midwifery in O ntario, C anada », Birth, vol. 18, no $2: 100-6$.

M ITFO R D, J. (1992) T heA merican W ay of Birth, N ew York, Dutton.

N EW FO U N DLAN D AN D LABR AD OR M IDW IVES'ASSO CIATION (1999, 3 mai). «C urrent midwifery situation in C anada », M idwifery in N ewfoundland and Labrador, Disponible: UR L : http:/ / www.ucs.mun.cal pherbert/ number8.html

ONTAR IO MINISTRY OF HEALTH (1987a) Report of the Task Force on the Implementation of M idwifery in 0 ntario », Toronto, Q ueen's Printers for 0 ntario, p. 195-232.

O N TAR IO M IN IST RY OF HEALT H (1987b) «A ppendix 1:A history of midwifery in C anada » dans R eport of the Task Force on the Implementation of M idwifery in 0 ntario », Toronto, Q ueen's Printers for O ntario, p. 195-232.

O R DR E DESSAGES-FEM M ES DE L'O N TAR IO (1994).D ocuments pour les sages-femmes, Toronto.

SAILLAN T, F. et M . O 'N EIL (dirs.) (1987). A ccoucher autrement, repères historiques, sodaux et aulturels de la grossesse et de l'accouchement au $Q$ uébec, M ontréal, Éd. Saint-M artin.

TY SO N , H., A. NIX O N , A.VAN DER SLO OT et K. HU GHES (1995). «T he re-emergence and professionalization of midwifery in $\mathrm{O}$ ntario, C anada », Issues in M idwifery, London, C Curchill, $\mathrm{p}$. 163-175. 